Check for updates

Cite this: RSC Adv., 2017, 7, 38243

\title{
Physicochemical properties and in vitro bioaccessibility of lutein loaded emulsions stabilized by corn fiber gums
}

\author{
Huimin Feng, ${ }^{a}$ Chao Li, (D) $\dagger^{\star a}$ Chin Ping Tan, ${ }^{b}$ Xiong Fu, ${ }^{a}$ Bin Zhang $\mathbb{D}^{a}$ \\ and Qiang Huang†^a
}

\begin{abstract}
Lutein is a natural colorant and functional ingredient with many health-promoting bioactivities. However, its use in functional foods is limited due to its low water-solubility and chemical instability. The objectives of this study were to investigate the physicochemical properties and in vitro bioaccessibilities of lutein loaded emulsions stabilized by corn fiber gums (CFG1 and CFG2). The physicochemical properties and stabilities of the emulsions were evaluated by droplet size and distribution, $\zeta$-potential, viscosity, visual observation of phase separation, and lutein retention during storage. The lutein bioaccessibilities of the emulsions were measured after digestion in a simulated gastrointestinal tract (SGT). The results showed that CFG2 had better emulsifying capacity and stability than CFG1. Compared to lutein dispersed in corn oil, the chemical stability and in vitro bioaccessibility of lutein in the emulsions were significantly increased through emulsification. These results suggest that corn fiber gum is a promising natural emulsifier, which has potential to fabricate emulsions for delivering lutein or other lipophilic bioactive

compounds in functional foods.
\end{abstract}

Received 2nd May 2017

Accepted 11th July 2017

DOI: $10.1039 / \mathrm{c} 7 \mathrm{ra04943a}$

rsc.li/rsc-advances

\section{Introduction}

Lutein is a natural coloring agent widely existing in many biological materials including yellow corn, egg yolk and marigold flowers. Lutein, belonging to the xanthophyll class of carotenoids, possesses many health-promoting bioactivities, such as lowering of the risk of age-related eye diseases and antioxidant activities. ${ }^{1-3}$ The use of lutein as a functional ingredient is currently limited due to its relatively low and variable oral bioavailability. ${ }^{1,4}$ Poor bioavailability is attributed to its chemical instability, low water-solubility and high melting point. ${ }^{5}$ Overcoming these limitations will broaden the application of lutein in functional foods and pharmaceutical fields. Oil-inwater $(\mathrm{O} / \mathrm{W})$ emulsion, consisting of small lipid droplets in an aqueous system, is a promising delivery system for improving the bioavailability of lipophilic bioactive compounds. ${ }^{4,6}$ For the emulsions, the lipid phase breaks down to form mixed micelles in the gastrointestinal tract. The lipid-dissolved lipophilic compounds are then solubilized in the mixed micelles, and thus increase their bioavailability., ${ }^{7,8}$ In addition, emulsion-based

${ }^{a}$ School of Food Science and Engineering, South China University of Technology, 381Wushan Road, Guangzhou, 510641, P. R. China. E-mail: lichao02263559@163. com; fechoh@scut.edu.cn; Fax: +86 20 87113845; Tel: +86 2087113845

${ }^{b}$ Department of Food Technology, Faculty of Food Science and Technology, Universiti Putra Malaysia, 43400 Serdang, Selangor, Malaysia

$\dagger$ Current address: School of Food Science and Engineering, South China University of Technology, 381 Wushan Road, Guangzhou 510640, China. delivery systems have been indicated to improve the physical and chemical stability of lutein. ${ }^{4,9}$ Nowadays, gum arabic (GA), one of the most commonly used emulsifiers, is extensively used to prepare $\mathrm{O} / \mathrm{W}$ emulsions due to its good emulsifying properties, low viscosity, and high water solubility. ${ }^{10,11}$ However, the utilization of GA has been restrained due to high cost and variable sources in the food industry. ${ }^{11,12}$ It was reported that increased utilization of natural emulsifiers in food products can result in a healthier and more sustainable food supply. ${ }^{13}$ Therefore, there is a need to find alternative natural polysaccharide-based emulsifiers with potential application within the food industry.

Corn is one of the most important economic crops in the world. According to the 2017 USDA's report, the amount of corn production in China will increase to more than two million tons. Corn fiber is considered as an abundant low-value by-product in the process of corn wet- and/or dry-milling. Corn fiber contains the most fibrous portions of corn kernel pericarp and endosperm tissues. Corn fiber gum (CFG) could be easily isolated from destarched and de-oiled corn fiber by the alkaline hydrogen peroxide extraction, which was proven to be a facile, reproducible, and economic method. ${ }^{14,15} \mathrm{CFG}$ has been demonstrated to have good thickening, emulsifying, and film-forming properties in a number of previous studies. ${ }^{15-18}$ Therefore, CFG has potential to be an ideal natural emulsifier for commercial utilization within the food industry. ${ }^{13}$ However, little work has been reported on the application of CFG in designing emulsion-based delivery systems for nutraceuticals, such as lutein. 
The aim of this work was therefore to study the physicochemical property and in vitro bioaccessibility of lutein loaded emulsions stabilized by corn fiber gum (CFG). Two CFG fractions (CFG1 and CFG2) were extracted from de-oiled and destarched corn fiber. The physicochemical properties and stabilities of lutein emulsions stabilized by CFG1, CFG2, and GA were comparatively evaluated by droplet size and distribution, zeta-potential, viscosity, visual observation of phase separation, and lutein retention during storage. Furthermore, in vitro bioaccessibilities of lutein in the emulsions were measured in a simulated gastrointestinal tract (SGT).

\section{Experimental}

\section{Materials and chemicals}

Corn fiber was kindly provided by Inner Mongolia Yuwang Biological Technology Co. Ltd (Tongliao, Inner Mongolia, China). This source of corn fiber used in this study has good reliability and no obvious batch-to-batch variations according to the manufacturer's instruction. The corn fiber was ground in a cutting mill and passed through a 20-mesh screen to obtain fine powder. Corn oil (Wilmar Trading Co. China) was purchased from a local store (Guangzhou, China) and used without further purification. Food grade $\alpha$-amylase (20000 U $\mathrm{mL}^{-1}$ ) was kindly provided by Novozymes Biotechnology Co. Ltd (Wuxi, China). Gum arabic (GA) (containing around 16.2\% moisture, $69.7 \%$ polysaccharide, $1.12 \%$ protein, and $0.3 \%$ minerals) was purchased from Cargill Co. (Minneapolis, USA). Trifluoroacetic acid (TFA), monosaccharide standards (arabinose; galactose; glucose; xylose; mannose) and uronic acid (galacturonic acid; glucuronic acid) were purchased from Aladdin Chemistry Co. (Shanghai, China). 2,2-diphenyl-1picrylhydrazyl (DPPH), gastric lipase $\left(10^{5}\right.$ units per $\left.\mathrm{g}\right)$, trypsin (300 units per $\mathrm{mg}$ ), pepsin (3000 units per $\mathrm{g}$ ), and bile salts were purchased from Sigma-Aldrich Chemical Co. (St. Louis, MO, USA). All other chemicals and solvents were of analytical grade.

\section{Extraction of corn fiber gum (CFG)}

CFG was isolated from de-oiled and de-starched corn fiber by the alkaline hydrogen peroxide method with modifications. ${ }^{\mathbf{1 4}}$ Corn fiber $(50.0 \mathrm{~g})$ was mechanically stirred into water $(0.5 \mathrm{~L})$ containing $\mathrm{NaOH}(2 \mathrm{~g})$ and $\mathrm{Ca}(\mathrm{OH})_{2}(1.9 \mathrm{~g})$. The mixture was boiled for $1 \mathrm{~h}$ and then centrifuged at $6000 \times g$ for $20 \mathrm{~min}$ and first supernatant was collected. The residue was resuspended in $400 \mathrm{~mL}$ of distilled water, boiling for $5 \mathrm{~min}$ and then centrifuged at $6000 \times g$ for $20 \mathrm{~min}$. The supernatant was collected and combined with first supernatant. Then $5.1 \mathrm{~g}$ of $30 \% \mathrm{H}_{2} \mathrm{O}_{2}$ was added to the combined extract (0.7 L, pH 11.3) and the $\mathrm{pH}$ was readjusted to 11.5 by adding $\mathrm{NaOH}$ solution (25\%) with stirring at room temperature for $2 \mathrm{~h}$. The $\mathrm{pH}$ was adjusted to $4.0-4.5$ by adding concentrated $\mathrm{HCl}$ to precipitate hemicellulose A, which was collected by centrifugation at $10000 \times g$ for $30 \mathrm{~min}$. Two volumes of ethanol were added to the supernatant to precipitate the major arabinoxylan fraction. Then the precipitate was collected by centrifugation at $5000 \times g$ for $10 \mathrm{~min}$ and lyophilized to obtain CFG-1. The residue left after alkali extraction was further extracted with alkaline $\mathrm{H}_{2} \mathrm{O}_{2}$ solution at $100{ }^{\circ} \mathrm{C}$ with stirring for $1.5 \mathrm{~h}$. The supernatant was separated by centrifugation at $6000 \times g$ for $15 \mathrm{~min}$ and then adjusted to $\mathrm{pH}$ 4.0-4.5. The precipitate was removed by centrifugation at $10000 \times g$ for 30 min. The CFG-2 was obtained from the supernatant by precipitating it with 2 -fold volumes of ethanol, collected and lyophilized.

\section{Chemical composition and structure analysis}

The carbohydrate content was determined by the phenolsulfuric acid colorimetric method. ${ }^{19}$ The protein content was determined by the Lowry's method..$^{20}$ The ferulic acid content was determined by a reported method. ${ }^{21}$

The molecular weight $\left(M_{\mathrm{w}}\right)$ distribution of the sample was measured using a reported method in our lab. ${ }^{22}$ In brief, the experiment was performed on an Agilent 1260 instrument (Agilent, USA) equipped with an Agilent 1260 Refractive Index Detector (Agilent Co., USA) and TSK-G5000 $(7.8 \times 300 \mathrm{~mm}$ i.d., $10 \mu \mathrm{m})$ and TSK-G3000 $(7.8 \times 300 \mathrm{~mm}$ i.d., $5 \mu \mathrm{m})$ analytical columns in series maintained at a temperature of $35{ }^{\circ} \mathrm{C}$. The columns were eluted with $0.02 \mathrm{M} \mathrm{KH}_{2} \mathrm{PO}_{4}$ buffer solution at

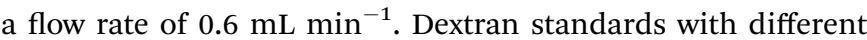
$M_{\mathrm{w}}(5.2,11.6,23.8,48.6,148,273,410,668$, and $1400 \mathrm{kDa})$ were used as standards. The calibration curve was obtained by plotting the retention time of standards against the logarithm of their respective $M_{\mathrm{w}}$.

The sample ( $4 \mathrm{mg}$ ) was hydrolyzed with $4 \mathrm{~mL}$ of TFA (4 M) at $110{ }^{\circ} \mathrm{C}$ for $6 \mathrm{~h}$ in a $10 \mathrm{~mL}$ ampere bottle. Excess TFA was removed using a rotary evaporator at $45{ }^{\circ} \mathrm{C}$. The residue was dissolved in $4 \mathrm{~mL}$ of methanol and evaporated to dryness using a nitrogen gas blowing instrument. The residue was redissolved in $8 \mathrm{~mL}$ of distilled water, filtered through a $0.22 \mu \mathrm{m}$ microfilter and then analyzed by an ion chromatography (IC) instrument (Dionex ICS 3000, Sunnyvale, CA). ${ }^{23}$ Standards of fucose, glucose, rhamnose, xylose, mannose, galactose, arabinose, glucuronic acid and galacturonic acid were used to determine the equation of the standard curve. The content of monosaccharide was calculated using a linear regression equation for each monosaccharide with various concentrations proportional to peak areas.

IR spectra of CFG1 and CFG2 were determined using an IR spectrophotometer (Vector 33, Bruker, Germany). The sample was mixed with $\mathrm{KBr}$ powder and pressed into a pellet for measurement. The spectrum of the sample was recorded in the range of $400-4000 \mathrm{~cm}^{-1}$.

\section{Interfacial tension measurement}

Interfacial tension of the sample was measured at different concentrations $(0,0.5$, and $2.0 \%)$ by the Wilhelmy plate method with a DCAT21 tensiometer (Datephysics CO., Germany). ${ }^{\mathbf{1 1}}$ Corn oil was added into a measuring cup and placed in a temperature-control jacket on a moveable platform. A platinum plate was immersed in corn oil by raising the platform. After lowering the platform, the measuring cup was removed. A constant volume of sample solution was added to another measuring cup. Immediately afterward, corn oil was gently layered on the 
top of sample solution. After equilibrium for $1 \mathrm{~min}$, the interfacial tension was measured.

\section{Preparation of lutein emulsion}

The formulation of emulsion was selected based on the result of optimization for preparing stable emulsions. The 5\% of CFG was the maximum level used in this study. Each aqueous phase was prepared by dispersing $5.0 \mathrm{~g}$ of emulsifier sample into $90 \mathrm{~mL}$ phosphate buffer ( $\mathrm{pH} 4.0)$ containing citric acid (0.13\%) and sodium benzoate $(0.12 \%)$. An organic phase was prepared by dissolving $25 \mathrm{mg}$ lutein $\left(250 \mathrm{mg} \mathrm{L}^{-1}\right.$ in the final emulsion) into $5 \mathrm{~g}$ corn oil. A coarse oil-in-water emulsion was prepared by mixing each aqueous phase and each organic phase using a high-speed shear homogenizer T25 (IKA Co., Germany) for $2 \mathrm{~min}$ at $13000 \mathrm{rpm}$. The resulting coarse emulsions were homogenized immediately using a single-pass laboratory-scale jet homogenizer M-110EH (MFIC Co., USA) three times at $50 \mathrm{MPa}$ to obtain fine emulsion samples.

\section{Droplet size and zeta-potential measurements}

The average droplet size and distribution of emulsion was measured using a Mastersizer analyzer (MS2000, Worcestershire, UK). The average droplet size of emulsion was characterized by the surface area mean diameter: $d_{3,2}=\sum n_{i} d_{i}{ }^{3} / \sum n_{i} d_{i}^{2}$, where $n_{i}$ is number of droplets, and $d_{i}$ is droplet diameter. The refractive indices of corn oil and buffer solution were 1.474 and 1.330 , respectively. The $d_{3,2}$ was manually calculated by data analysis software.

The $\zeta$-potential of emulsion droplets was measured using a Zetasizer Nano-ZS instrument (Malvern Instruments, Worcestershire, U.K.) equipped with a MPT-2 $\mathrm{pH}$ autotitrator at room temperature. The diluted emulsions $(1: 10)$ were injected directly into the capillary electrophoresis cell and then inserted into the module. The $\zeta$-potential was automatically calculated by measuring the electrophoresis mobility of the droplets.

\section{Viscosity measurement}

Viscosity of fresh emulsion was measured using a NDJ-8S digital viscometer (Shanghai Rex Instruments, China) at room temperature. The first rotator was selected and rotation speed was set at $60 \mathrm{rpm}$.

\section{Encapsulation efficiency of lutein measurement}

The $50 \mu \mathrm{L}$ of emulsion sample was mixed with $4.95 \mathrm{~mL}$ of DMSO in a brown bottle and incubated at $40{ }^{\circ} \mathrm{C}$ for $1 \mathrm{~h}$ with ultrasonic treatment. The resulting mixture was centrifugated at $3500 \mathrm{rpm}$ for $30 \mathrm{~min}$ and the supernatant was collected. The lutein concentration in the supernatant was determined by absorbance measurements at $446 \mathrm{~nm}$ according to a previous method. ${ }^{4}$ The encapsulation efficiency (EE) of lutein in the emulsion sample was calculated using the eqn (1):

$$
\begin{gathered}
\mathrm{EE}(\%)=\underset{\text { lutein content of supernatant/lutein content of }}{\text { emulsion }} \\
\text { (1) }
\end{gathered}
$$

\section{In vitro digestion assay}

The in vitro digestion of lutein-enriched emulsions in a SGT model was carried out according to the method described previously. ${ }^{11}$ Briefly, emulsion sample $(1.5 \mathrm{~mL})$ was mixed with $13.5 \mathrm{~mL}$ of saline (containing $140 \mathrm{mM} \mathrm{NaCl}, 5 \mathrm{mM} \mathrm{KCl}$, and $150 \mu \mathrm{M}$ BHT) and stirred (500 rpm) for 10 min using a magnetic stirrer. The mixture was incubated at $37^{\circ} \mathrm{C}$ and acidified to $\mathrm{pH}$ 1.2 with $1.0 \mathrm{M} \mathrm{HCl}$ solution. The resulting mixture was mixed with $1 \mathrm{~mL}$ of pepsin solution $\left(40 \mathrm{mg} \mathrm{mL}^{-1}\right)$ and incubated at $37^{\circ} \mathrm{C}$ under magnetic stirring (500 rpm) for $1 \mathrm{~h}$. After gastric digestion, the $\mathrm{pH}$ of the digestive sample was adjusted to 6.8 with $1.0 \mathrm{M} \mathrm{NaOH}$ and $4.5 \mathrm{~mL}$ of intestinal enzymes (including $2 \mathrm{mg} \mathrm{mL}^{-1}$ of pancreatin and $12 \mathrm{mg} \mathrm{mL}^{-1}$ of porcine bile extract prepared with $0.1 \mathrm{M} \mathrm{NaHCO}_{3}$ ) were added to start the digestion process, which occurred in a shaking water bath at $37^{\circ} \mathrm{C}$ for $2 \mathrm{~h}$. During lipolysis, the $\mathrm{pH}$ was maintained at $6.8 \pm 0.02$ by adding $0.2 \mathrm{M} \mathrm{NaOH}$ manually. The amount of $\mathrm{NaOH}$ consumed over time was recorded through intestinal ingestion to calculate the concentration of free fatty acids (FFA). Raw corn oil containing the same concentration of lutein as the emulsion was used as the control. The amount of FFA was calculated using the eqn (2):

$$
\text { FFA }(\%)=\frac{V_{\mathrm{NaOH}} \times m_{\mathrm{NaOH}} \times M_{\text {lipid }}}{W_{\text {lipid }} \times 2} \times 100
$$

where $V_{\mathrm{NaOH}}$ was the volume of $\mathrm{NaOH}$ required to neutralize the released FFA (L), $m_{\mathrm{NaOH}}$ was the molarity of $\mathrm{NaOH}$ (in M), $W_{\text {lipid }}$ was the total mass of corn oil initially present in the digestion emulsion ( $\mathrm{g}$ ), and $M_{\text {lipid }}$ was average molecular mass of corn oil $\left(872 \mathrm{~g} \mathrm{~mL}^{-1}\right)$.

\section{Determination of lutein bioaccessibility}

The in vitro bioaccessibility of lutein was determined using the procedure described previously with a few modifications. ${ }^{10}$ In brief, the above end-product of digestion in the simulated gastrointestinal tract was centrifuged to obtain three phases (top, middle and bottom). The middle aqueous phase was filtered through a $0.22 \mu \mathrm{m}$ microfilter (Millipore corp., Bedford, $\mathrm{MA}$ ), and the concentration of lutein in the aqueous phase was determined by absorbance measurements $(446 \mathrm{~nm})$ according to a previous method. ${ }^{4}$ The bioaccessibility of lutein after digestion was calculated using the eqn (3):

$$
\text { Bioaccessibility }(\%)=\frac{V_{\text {aqueous }} \times C_{\text {aqueous }}}{W_{\text {lipid }}}
$$

where $V_{\text {aqueous }}$ was the volume of the aqueous phase, $C_{\text {aqueous }}$ was the concentration of lutein (not including chemical transformation of lutein) in the aqueous phase, and $W_{\text {lipid }}$ was the mass of the lutein in corn oil, respectively.

\section{Physical stability analysis}

The physical stability of emulsion was evaluated by changes of droplet size and creaming index after seven days of storage. Fresh emulsion samples were transferred to glass tubes sealed with screw caps to prevent evaporation, and stored at room temperature for 7 days. During storage, the extent of creaming was characterized by the creaming index (\%), which is defined 
as creaming index $=\left(H_{\mathrm{S}} / H_{\mathrm{E}}\right) \times 100 \%$, where $H_{\mathrm{S}}$ is the height of the serum layer and $H_{\mathrm{E}}$ is the initial emulsion height. The change in $d_{3,2}$ was measured using a Mastersizer analyzer.

\section{Chemical stability analysis}

The chemical stability of lutein emulsions was evaluated by lutein retention in emulsions under different storage conditions. The temperature and light had no significant effects on particle size and creaming stability of the emulsions. The changes of particle size and creaming index were similar with above results of physical stability (data not shown). For thermal stability analysis, $10 \mathrm{~mL}$ of fresh emulsion samples were transferred into $25 \mathrm{~mL}$ screw-cap bottles and stored in the dark at 25 and $55{ }^{\circ} \mathrm{C}$ for a period of $7 \mathrm{~d}$. Another group of emulsions were stored in the dark or light at $25{ }^{\circ} \mathrm{C}$ for a period of $7 \mathrm{~d}$. Changes of lutein content were measured every two days according to the method described previously. ${ }^{4}$

\section{Results and discussion}

\section{Chemical composition and preliminary structure}

The chemical compositions of CFG1 and CFG2 are shown in Table 1 . The yields of CFG1 and CFG2 based on original corn fiber were 16.6 and $5.7 \%$, respectively. The moisture contents of CFG1 and CFG2 were 9.66 and $11.3 \%$, respectively. CFG1 contained carbohydrate $(88.9 \%)$, protein $(0.27 \%)$, and ferulic acid $(0.45 \%)$, respectively, while CFG2 consisted of carbohydrate $(85.6 \%)$, protein $(1.0 \%)$, and ferulic acid (1.2\%), respectively.

The monosaccharide compositions of CFG1 and CFG2 as determined by ion-exchange chromatography are shown in Fig. 1. CFG1 and CFG2 were composed of Ara, Gal, Glu, Xyl, Man, GalA, and GlcA with similar molar ratios. According to the linear regression equation, CFG1 was composed of Ara, Gal, Glu, Xyl, Man, GalA, and GlcA in molar percentages of 24.4, 11.4, 3.97, 35.9, 0.16, 19.8, and 4.37\%, respectively, and CFG2 consisted of Ara, Gal, Glu, Xyl, GalA, and GlcA in molar percentages of $25.0,11.4,6.58,34.5,1.40,18.1$, and $3.02 \%$,

Table 1 Chemical compositions and structure of CFG1 and CFG2 from corn fiber

\begin{tabular}{lll}
\hline Item & CFG1 & CFG2 \\
\hline Yield (\%) & $16.6 \pm 0.3$ & $5.7 \pm 0.1$ \\
Moisture (\%) & $9.66 \pm 0.32$ & $11.3 \pm 0.15$ \\
Carbohydrate (\%) & $88.9 \pm 0.87$ & $85.6 \pm 3.9$ \\
Protein (\%) & 0.27 & 1.0 \\
Ferulic acid (\%) & 0.45 & 1.2
\end{tabular}

Monosaccharide compositions (molar percentage)

$\begin{array}{lll}\text { Ara (\%) } & 24.4 & 25.0 \\ \text { Gal (\%) } & 11.4 & 11.4 \\ \text { Glc (\%) } & 3.97 & 6.58 \\ \text { Xyl (\%) } & 35.9 & 34.5 \\ \text { Man (\%) } & 0.16 & 1.40 \\ \text { GalA (\%) } & 19.8 & 18.1 \\ \text { GlcA (\%) } & 4.37 & 3.02 \\ \text { Molecular weight (kDa) } & 319.2 & 382.8\end{array}$

respectively (Table 1). Previous studies reported that CFG was a high molecular-weight hydrophilic biopolymer composed of D-xylose (48-54\%), L-arabinose (33-35\%), galactose (7-11\%), and glucuronic acid (3-6\%). ${ }^{\mathbf{1 4 2 4}}$ The result showed that CFG1 and CFG2 had different chemical compositions from previous reports.

The molecular weights of CFG1 and CFG2 were determined using HPGPC as shown in Fig. 2A. The regression equation of dextran standards was $\log M_{\mathrm{w}}=-0.2025 X+9.8398$ (where $M_{\mathrm{w}}$ represents the molecular weight of dextrans and $X$ represents the retention time) with a correlation coefficient of 0.999 . Both CFG1 and CFG2 exhibited a single and symmetrical elution peak, indicating their homogeneity. According to the equation and retention time, the average molecular weights of CFG1 and CFG2 were calculated to be 319.2 and $382.8 \mathrm{kDa}$, respectively (Table 1).

IR analysis has been widely adopted to elucidate the structure and configuration of polysaccharides. ${ }^{22}$ As shown in Fig. 2B, the broad absorption peaks at $3400 \mathrm{~cm}^{-1}$ and weak absorption peaks at around $2927 \mathrm{~cm}^{-1}$ were the characteristic stretching peaks of $\mathrm{O}-\mathrm{H}$ and $\mathrm{C}-\mathrm{H}$, respectively. The peaks at $1040 \mathrm{~cm}^{-1}$ were ascribed to the stretching vibration of $\mathrm{C}-\mathrm{O}-\mathrm{C}$ in xylopyranose rings. ${ }^{25}$ The stretching peaks at $900 \mathrm{~cm}^{-1}$ were the characteristic of $\beta$-D-pyranosidic configuration. ${ }^{12}$

\section{Interfacial tension}

In order to understand the interfacial characteristics of the samples, interfacial tensions of CFG1, CFG2 and GA solutions were measured. As shown in Fig. 3A, the interfacial tensions of the samples decreased with the increase in the concentrations from 0.1 to $2.0 \%$. However, three samples showed significant differences to lower the interfacial tension. At the concentration of $2.0 \%$, the interfacial tension values of CFG1, CFG2, and GA solutions were $20.8,17.8$, and $19.1 \mathrm{mN} \mathrm{m}^{-1}$, respectively. Interfacial characteristic of surface-active ingredient could influence the formation and stabilities of the emulsions. ${ }^{17}$ CFG2 with lower interfacial tension values resulted in the formation of smaller droplets during emulsification, thus forming a more stable emulsion. ${ }^{21,26}$ It is known that polysaccharide-based emulsifiers are less effective at reducing the interfacial tension than small molecular surfactant or proteins. ${ }^{17}$ The protein and ferulic acid played a main role in reducing the interfacial tension in the oil-water interface. Therefore, lower interfacial tension of CFG2 solution might be due to the fact that CFG2 contained more protein and ferulic acid. Overall, these results indicated that CFG2 was more effective than CFG1 and GA in reducing the interfacial tension of corn oil-water interface.

\section{Characterization of the emulsions}

In order to compare the emulsifying properties of CFG1, CFG2, and GA, the formation of lutein loaded oil-in-water emulsions were initially investigated. Fig. 3B shows the droplet size distributions of three emulsions. Compared to CFG1-stabilized emulsion, CFG2-stabilized emulsion had a narrower droplet size distribution, ranging from $0.02-2.0 \mu \mathrm{m}$. The average $d_{3,2}$ 

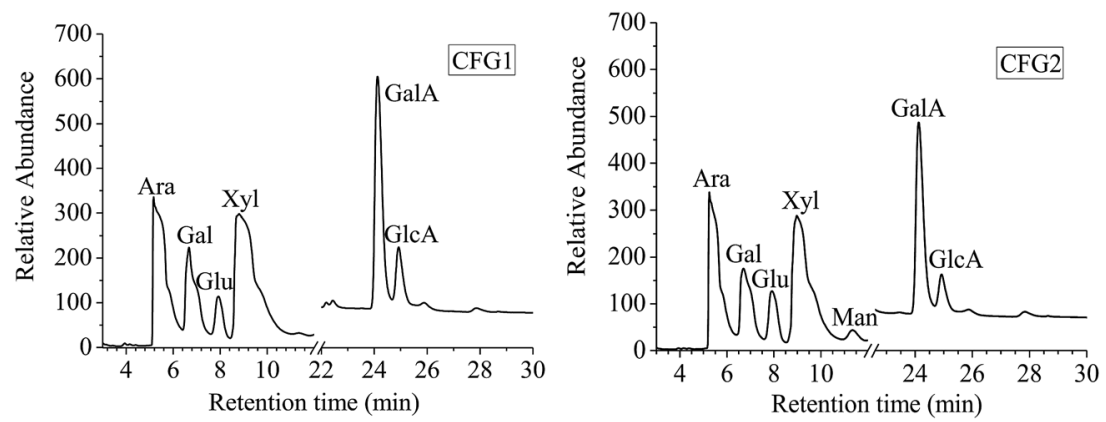

Fig. 1 Monosaccharide composition IC profiles of CFG1 and CFG2.

values of the emulsions stabilized by CFG1 and CFG2 were 205 and $162 \mathrm{~nm}$, respectively. It is well known that the droplet size of $\mathrm{O} / \mathrm{W}$ emulsion depends on two factors, including the initial formation of droplets diameter and the rapid stabilization of droplets by emulsifiers against bridging flocculation and recoalescence. ${ }^{17}$ Thus, the final droplet size depends on droplet disruption ability and adsorption rate onto the surface of dispersed oil droplets of emulsifiers. The proteinaceous and ferulic acid moieties have been reported to facilitate the adsorption of CFG onto the surface of dispersed oil droplets. ${ }^{13-15,21}$ Similar results could be found in other polysaccharide-based emulsifiers, such as beet pectin and gum arabic. ${ }^{21,27}$ Therefore, high contents of protein and ferulic acid of CFG2 might explain its good emulsifying property.

$\zeta$-potential is an important indication of electrostatic repulsion force. Droplets with higher absolute $\zeta$-potential can reduce aggregation and flocculation of the droplets, which was more favorable for the emulsion stability. ${ }^{27,28}$ The $\zeta$-potentials of lutein loaded emulsions are shown Fig. 3C. The $\zeta$-potential values of three emulsions were $-5.86,-8.23$, and $-10.6 \mathrm{mV}$, respectively. CFG2-stabilized emulsion had higher absolute $\zeta$-potential, indicating that the emulsion was more stable. ${ }^{29} \mathrm{In}$ addition, CFG1 and CFG2 were indicated as anionic emulsifiers, which were due to the existence of ferulic acid in their molecular chain. ${ }^{15,30}$

Fig. 3D shows the viscosity of lutein emulsions stabilized by CFG1, CFG2 and GA at the same concentration. The emulsion stabilized by CFG2 had higher apparent viscosity than CFG1 and GA. The ability of polysaccharides to increase viscosity depends on their molecular weight, conformation and interactions. Increasing molecular weight and radius of hydration could increase the viscosity of polymer solution. ${ }^{17,31}$ Therefore, differences in apparent viscosities of CFG1 and CFG2 might be caused by their different molecule weight and structure.

\section{In vitro digestion and bioaccessibility of lutein emulsions}

To investigate the effects of CFG1, CFG2 and GA on the lipid digestion and bioaccessibility of lipid-soluble lutein, corn oil and three lutein-enriched emulsions were digested in the SGT. Fig. 4A showed different digestion profiles in four formulations. The amount of FFA released increased with increasing of digestion time during the first $100 \mathrm{~min}$, at which point the FFA reached a plateau level. The final amount of FFA release decreased in the order of CFG2 $(90.9 \%)>$ CFG1 $(82.7 \%)>$ GA $(74.4 \%)>$ corn oil $(26.9 \%)$. In addition, the rate of FFA release also decreased in the order of CFG2 $>$ CFG1 $>$ GA $>$ corn oil. The lower rate and extent of bulk oil (corn oil) was mainly attributed to the low reactive area available for lipase adsorption due to the large oil droplet size. ${ }^{\mathbf{8 , 1 0 , 3 2}}$ Thus, CFG2stabilized emulsion exhibited the maximum rate and extent of FFA release.

After lipid digestion, the lipid was hydrolyzed into diacylglycerols and monoglycerols. The FFA released from the interface of oil droplets will combine with bile salts and phospholipids to form micelles, in which lutein dissolved in lipid could be solubilized and thus become bioaccessible. ${ }^{33,34}$ As
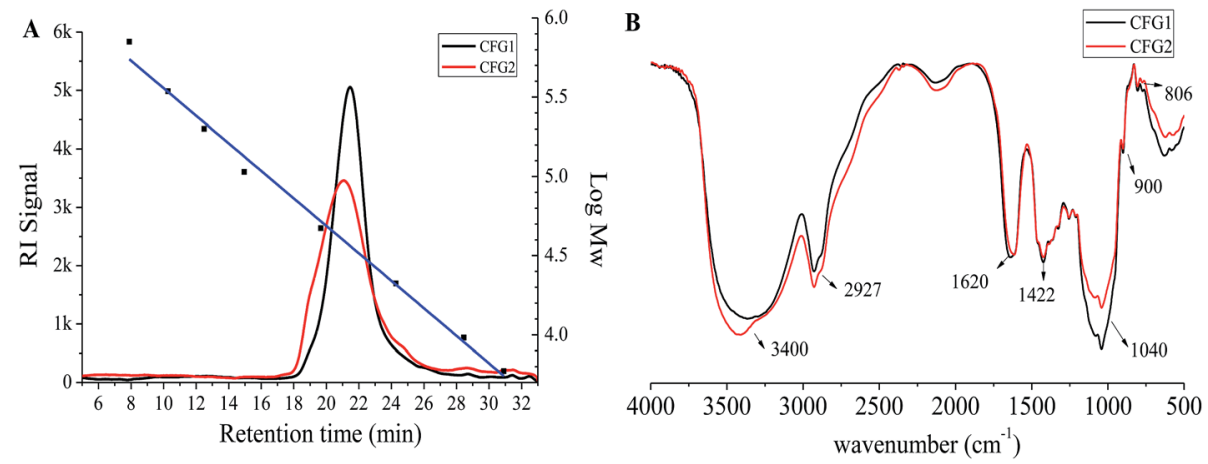

Fig. $2 M_{w}$ distributions (A) and IR spectra (B) of CFG1 and CFG2. 

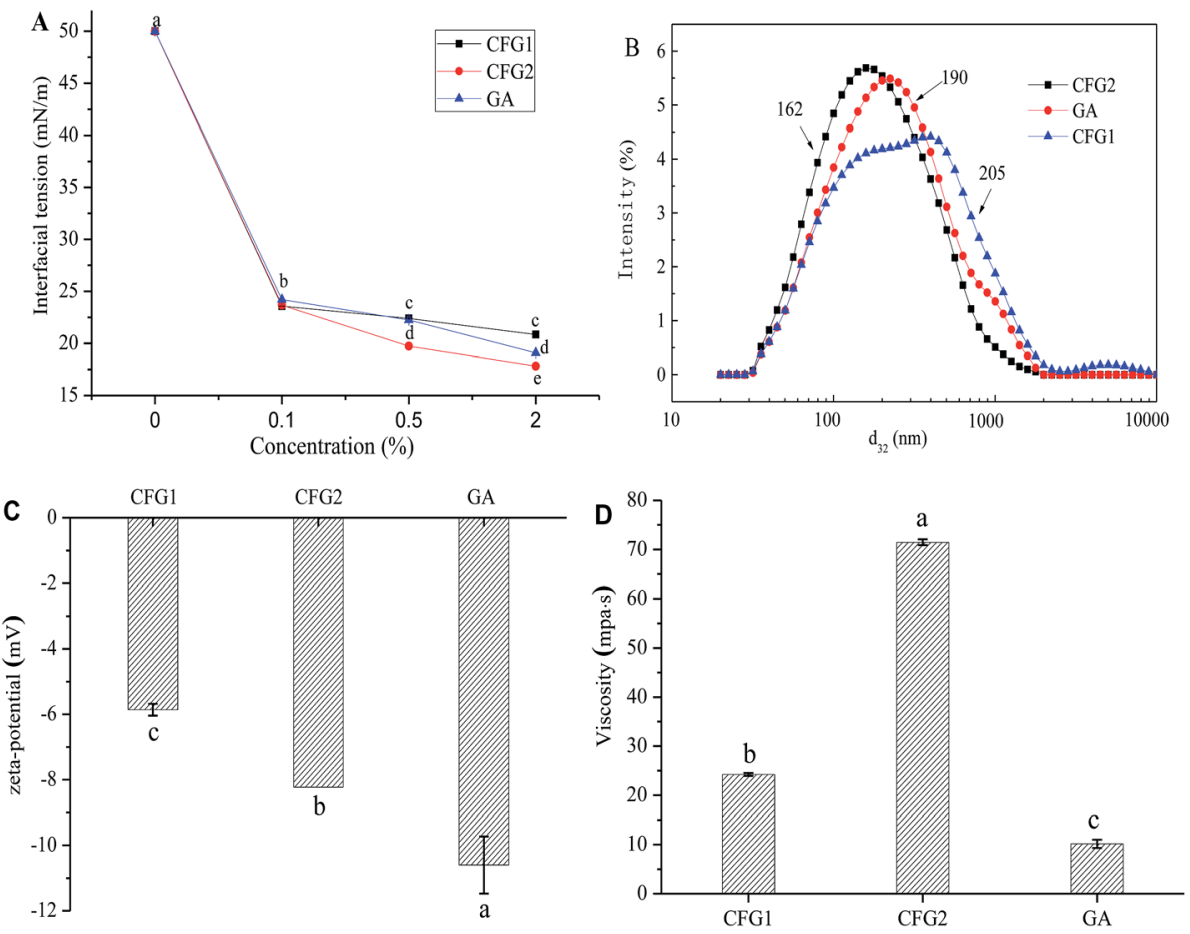

Fig. 3 (A) Influence of CFG1, CFG2 and GA concentrations on the interfacial tension at the corn oil-water interface; (B) droplet size distribution and (C) $\zeta$-potential values of emulsions stabilized by CFG1, CFG2 and GA, respectively; (D) viscosity of fresh prepared emulsions. Data with different letters are significantly different $(p<0.05)$.

shown in Fig. 4B, lutein bioaccessibilities of emulsions were greatly improved compared to that of corn oil. The encapsulation efficiency of lutein in CFG1-, CFG2-, and GA-stabilized emulsions were 79,86 , and $88 \%$, respectively. Based on the results of encapsulation efficiency of lutein, the bioaccessibility of lutein decreased in the order CFG2 $(32.4 \%)>$ GA $(27.7 \%)>$ CFG1 $(24.9 \%)>$ corn oil $(13.8 \%)$, indicating that an increase in the extent of lipolysis would improve bioaccessibility similar results were found in the reported studies., ${ }^{\mathbf{8} 32}$ The results demonstrated that corn fiber gum-stabilized emulsion could be used as a delivery system for improving the bioavailability of lutein. It has been reported that $10 \mathrm{mg}$ per day of lutein is an effective dose for providing protection against diseases..$^{35}$ Based on the above results, the lowest effective dosage of lutein in the emulsions absorbed by human body was calculated to be
Table 2 Changes in $d_{3,2}$ and creaming index of the emulsions stabilized by CFG1, CFG2 and GA ${ }^{a}$

\begin{tabular}{llllll}
\hline & \multicolumn{2}{l}{ Fresh emulsions } & & \multicolumn{2}{l}{ After 7 d of storage } \\
\cline { 2 - 3 } \cline { 5 - 6 } Sample & $d_{3,2}(\mathrm{~nm})$ & $\mathrm{CI}(\%)$ & & $d_{3,2}(\mathrm{~nm})$ & CI $(\%)$ \\
\hline CFG1 & $205^{\mathrm{a}}$ & $0^{\mathrm{a}}$ & & $205 \pm 1.0^{\mathrm{a}}$ & $7.0 \pm 1.0^{\mathrm{a}}$ \\
CFG2 & $162^{\mathrm{b}}$ & $0^{\mathrm{a}}$ & & $172 \pm 2.3^{\mathrm{b}}$ & $0^{\mathrm{b}}$ \\
GA & $190 \pm 0.5^{\mathrm{c}}$ & $0^{\mathrm{a}}$ & & $212 \pm 2.0^{\mathrm{c}}$ & $3.0^{\mathrm{c}}$
\end{tabular}

${ }^{a}$ Data in columns with different superscript letters are significantly different $(p<0.05)$.

$>49.2 \mathrm{mg} \mathrm{mL}{ }^{-1}$. Therefore, CFG-stabilized lutein emulsions could meet the demand for effective dosage of lutein in commercial products.
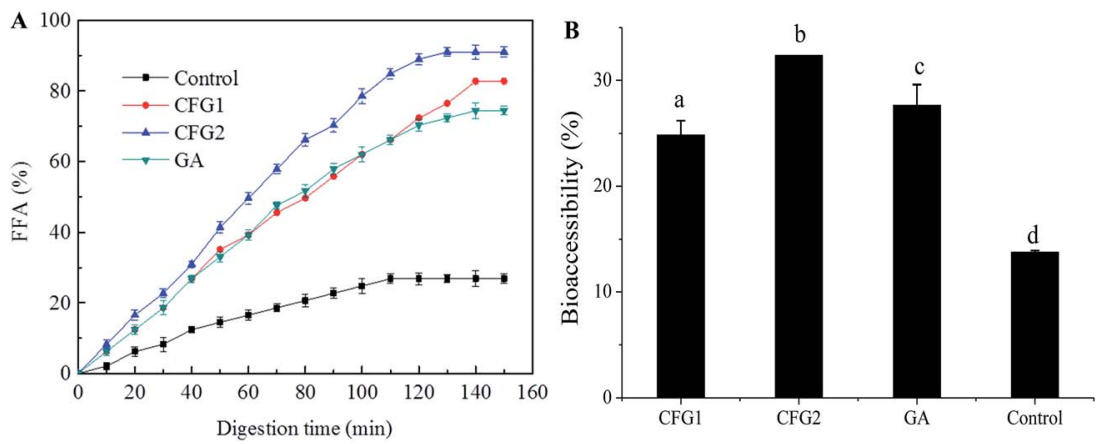

Fig. 4 (A) Release profiles of FFA released from corn oil and emulsions in the digestion process; (B) bioaccessibilities of lutein in three emulsions and bulk oil (corn oil) after in vitro lipolysis. Data with different letters are significantly different $(p<0.05)$. 


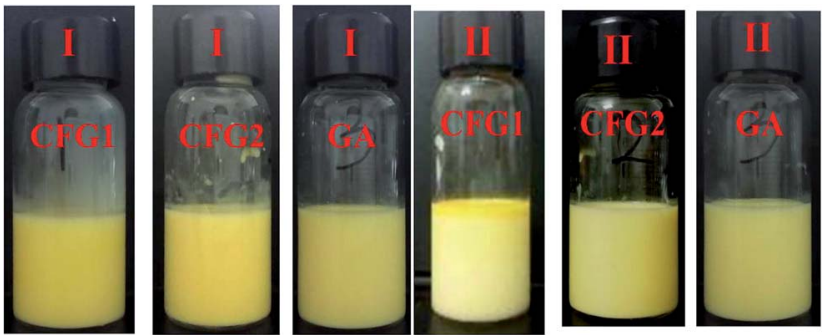

Fig. 5 Optical photographs of the emulsions stabilized by CFG1 CFG2, and GA, respectively. (I) Fresh emulsions; (II) the emulsions after storage for $7 \mathrm{~d}$ at room temperature.

\section{Physical stability of lutein emulsions}

The changes in $d_{3,2}$ and creaming index of the emulsions are shown in Table 2. Moreover, to visually observe the creaming, optical photographs were captured. As shown in Fig. 5, fresh lutein emulsions exhibited more stability, and no creaming was appeared. After 7 days of storage, the CFG2-stabilized emulsion kept good stability. The $d_{3,2}$ increased slightly from 162 to $172 \mathrm{~nm}$. However, a different extent of creaming were observed in the CFG1- and GA-stabilized emulsions, with a creaming index of $7 \%$ and $3 \%$, respectively (Table 2 ). The $d_{3,2}$ of the emulsion stabilized by CFG1 remained the same, whereas the $d_{3,2}$ of the emulsion stabilized by GA slightly increased from 190 to $212 \mathrm{~nm}$. Droplet size and viscosity greatly influenced the creaming velocity of emulsion, which can be explained by Stokes' Law. ${ }^{36}$ Higher viscosity of the emulsion stabilized by
CFG2 (71.5 MPa s) could also explain higher emulsion stability. This phenomenon can be supported by the previous study that higher viscosity could improve the emulsion stability through retarding free motion of droplets. ${ }^{22}$ However, these emulsions with low absolute $\zeta$-potential values exhibited excellent stability after 7 days of storage, which was due to that the electrostatic repulsive force was not the main stabilization mechanism for this emulsions. Previous studies have reported that polysaccharide-based emulsifiers, such as gum arabic, corn fiber gum, and beet pectin, favor the emulsion stability mainly through thickening and steric repulsion generated by their molecular structure. ${ }^{\mathbf{1 1 , 1 7}}$ In all, this result indicated that CFG has better stabilizing capacity than GA.

\section{Chemical stability of emulsion loaded with lutein}

In the present of heat, light, and oxygen, lutein is readily prone to isomerization and chemical degradation. ${ }^{37}$ We therefore monitored the chemical stability of lutein in the emulsion by measuring the degradation extent of lutein during different storage conditions.

As shown in Fig. 6, after storage at 25 and $55^{\circ} \mathrm{C}$ in the dark or in the light for a period of time, the lutein degradation of the emulsions increased with the increase in the storage time but in a slower speed compared to the control. In addition, the degradation rates of lutein increased with the increase in the storage temperature, and in the present of/irradiation by light, the lightness could also accelerate the degradation of lutein. This result further explained the change of color from yellow to
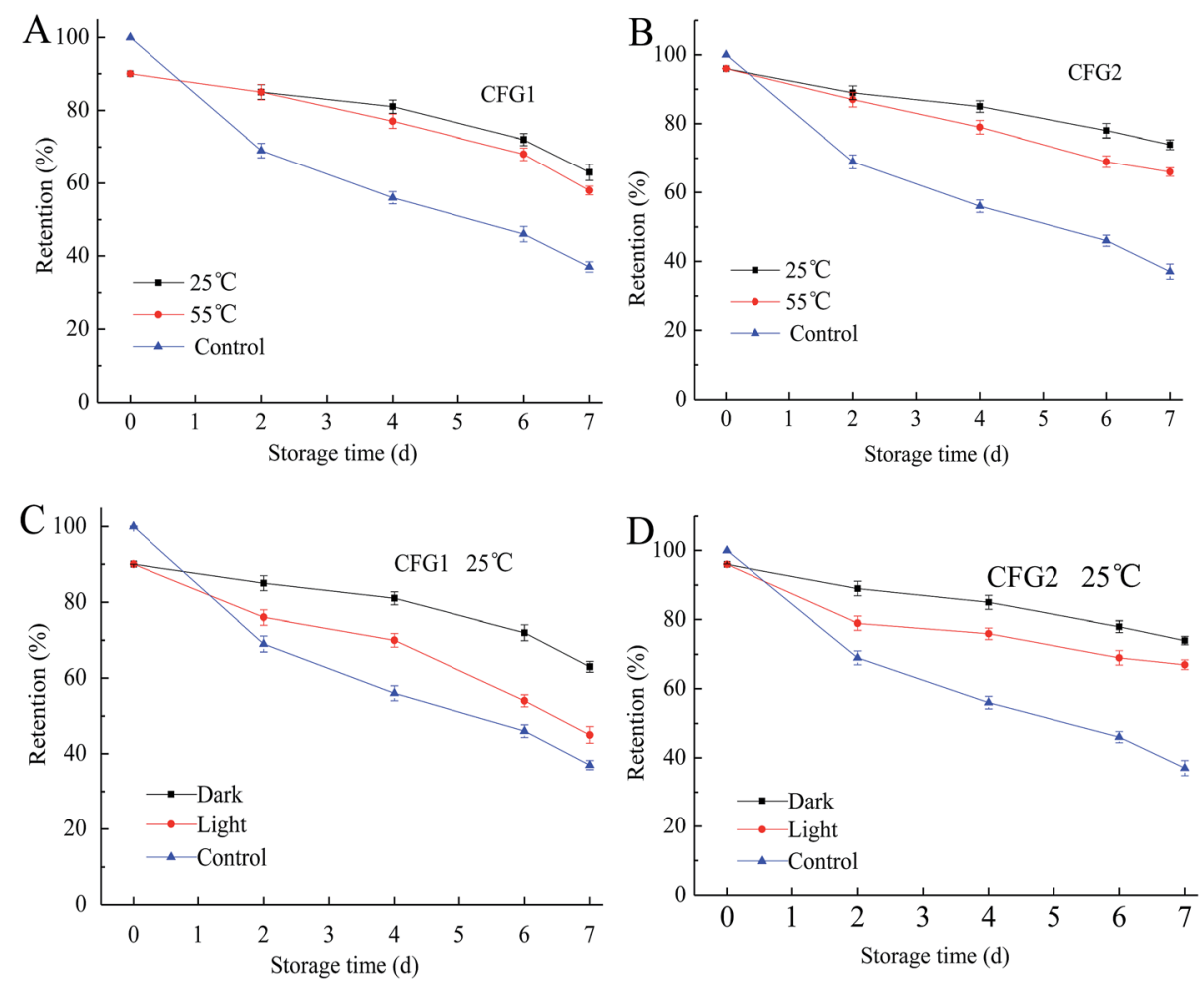

Fig. 6 Degradation of lutein in emulsions stabilized by CFG1 and CFG2 under different storage conditions for $7 \mathrm{~d}$. Lutein in the bulk oil was used as a control. 
light yellow in the CFG1-, CFG2-, and GA-stabilized emulsions. Similar result was found in the reported study that lutein was prone to degrade at higher temperatures or in the light. ${ }^{4}$ However, lutein of the emulsions stabilized by CFG2 and CFG1 showed higher stabilities than GA. These results indicated that CFG-stabilized emulsion was capable of inhibiting deterioration of lutein. Taken together, CFG revealed better emulsifying properties and improved physical and chemical stabilities of lutein emulsions when compared to a commercial emulsifier (GA).

\section{Conclusion}

In this study, CFG1 and CFG2 were isolated from corn fiber by the alkaline hydrogen peroxide method. CFG1 contained $88.9 \%$ of carbohydrate, $0.27 \%$ of protein, and $0.45 \%$ of ferulic acid, while CFG2 contained $85.6 \%$ of carbohydrate, $1.0 \%$ of protein, and $1.2 \%$ of ferulic acid. CFG1 and CFG2 had similar monosaccharide compositions but different from previous reports. ${ }^{\mathbf{1 4 2 4}}$ CFG2 exhibited better emulsifying capacity and emulsification stability than CFG1 and GA. The lutein bioaccessibilities of the emulsions stabilized by CFG1 and CFG2 were increased from $13.8 \%$ (corn oil) to $24.9 \%$ and $32.4 \%$, respectively. During storage, CFG1- and CFG2-stabilized emulsions could significantly inhibit the degradation of lutein under the conditions of high temperature and the light. These results suggest that corn fiber gum could be used to fabricate emulsions for delivering lutein or other lipophilic bioactive compounds. Thus, this research contributes to the development and utilization of this natural emulsifier in the food industry.

\section{Acknowledgements}

This work was funded by the China Postdoctoral Science Foundation (2016M602474), Science and Technology Planning Project of Nansha, Guangzhou (2016GJ001) and the Fundamental Research Funds for the Central Universities of China (2017BQ100).

\section{References}

1 J. Mares, Annu. Rev. Nutr., 2016, 36, 571-602.

2 B. Nidhi, G. Sharavana, T. R. Ramaprasad and B. Vallikannan, Food Funct., 2015, 6, 450-460.

3 T. T. Y. Woo, S.-Y. Li, W. W. K. Lai, D. Wong and A. C. Y. Lo, Graefe's Arch. Clin. Exp. Ophthalmol., 2013, 251, 41-51.

4 G. Davidov-Pardo, C. E. Gumus and D. J. McClements, Food Chem., 2016, 196, 821-827.

5 D. J. McClements and Y. Li, Adv. Colloid Interface Sci., 2010, 159, 213-228.

6 D. J. McClements, E. A. Decker and J. Weiss, J. Food Sci., 2007, 72, R109-R124.

7 A. Nagao, Jpn. Agric. Res. Q., 2014, 48, 385-391.

8 J. Xiao, C. Li and Q. Huang, J. Agric. Food Chem., 2015, 63, 10263-10270.

9 C. E. Gumus, G. Davidov-Pardo and D. J. McClements, Food Hydrocolloids, 2016, 60, 38-49.
10 P. S. Given, Curr. Opin. Colloid Interface Sci., 2009, 14, 43-47. 11 V. Castel, A. C. Rubiolo and C. R. Carrara, Food Hydrocolloids, 2017, 63, 170-177.

12 S. Patel and A. Goyal, Int. J. Food Prop., 2015, 18, 986-998.

13 D. J. McClements, L. Bai and C. Chung, Annu. Rev. Food Sci. Technol., 2017, 8, 205-236.

14 M. P. Yadav, D. B. Johnston, A. T. Hotchkiss Jr and K. B. Hicks, Food Hydrocolloids, 2007, 21, 1022-1030.

15 S. Kokubun, M. P. Yadav, R. A. Moreau and P. A. Williams, Food Hydrocolloids, 2014, 41, 164-168.

16 K. S. Mikkonen, M. P. Yadav, P. Cooke, S. Willför, K. B. Hicks and M. Tenkanen, BioResources, 2008, 3, 178-191.

17 L. Bai, S. Huan, Z. Li and D. J. McClements, Food Hydrocolloids, 2017, 66, 144-153.

18 S. Qiu, M. P. Yadav, H. Chen, Y. Liu, E. Tatsumi and L. Yin, Carbohydr. Polym., 2015, 115, 246-252.

19 M. Dubois, K. A. Gilles, J. K. Hamilton, P. Rebers and F. Smith, Anal. Chem., 1956, 28, 350-356.

20 O. H. Lowry, N. J. Rosebrough, A. L. Farr and R. J. Randall, J. Biol. Chem., 1951, 193, 265-275.

21 E. N. Fissore, A. M. Rojas, L. N. Gerschenson and P. A. Williams, Food Hydrocolloids, 2013, 31, 172-182.

22 C. Li, X. Fu, F. Luo and Q. Huang, Food Hydrocolloids, 2013, 32, 79-86.

23 C. Li, X. Li, L. You, X. Fu and R. H. Liu, Carbohydr. Polym., 2017, 155, 261-270.

24 M. P. Yadav, M. L. Fishman, H. K. Chau, D. B. Johnston and K. B. Hicks, Cereal Chem., 2007, 84, 175-180.

25 R. C. Sun and X. F. Sun, Carbohydr. Polym., 2002, 49, 415423.

26 Y. Zhang, C. Tan, S. Abbas, K. Eric, S. Xia and X. Zhang, Food Hydrocolloids, 2015, 51, 108-117.

27 H.-m. Chen, X. Fu and Z.-g. Luo, Food Hydrocolloids, 2016, 54, 99-106.

28 P. Shao, Y. Zhu and W. Jin, Food Hydrocolloids, 2017, 64, 2835.

29 Y. Yamanaka, I. Kobayashi, M. A. Neves, S. Ichikawa, K. Uemura and M. Nakajima, LWT-Food Sci. Technol., 2017, 76, 344-350.

30 J. Cirre, S. Al-Assaf, G. O. Phillips, M. P. Yadav and K. B. Hicks, Food Hydrocolloids, 2014, 35, 122-128.

31 X. Yu, C. Zhou, H. Yang, X. Huang, H. Ma, X. Qin and J. Hu, Carbohydr. Polym., 2015, 117, 650-656.

32 L. Salvia-Trujillo, C. Qian, O. Martín-Belloso and D. J. McClements, Food Chem., 2013, 141, 1472-1480.

33 R. Liang, C. F. Shoemaker, X. Yang, F. Zhong and Q. Huang, J. Agric. Food Chem., 2013, 61, 1249-1257.

34 Y. Ting, Y. Jiang, C.-T. Ho and Q. Huang, J. Funct. Foods, 2014, 7, 112-128.

35 K. Frede, A. Henze, M. Khalil, S. Baldermann, F. J. Schweigert and H. Rawel, J. Funct. Foods, 2014, 8, 118127.

36 M. N. Nasrabadi and S. A. H. Goli, Food Chem., 2016, 199, 258-264.

37 M. M. Alam, K. Ushiyama and K. Aramaki, J. Oleo Sci., 2009, 58, 361-367. 